\title{
Metastatic mechanism of spermatic cord tumor from stomach cancer
}

\author{
Masahiro Seike $\cdot$ Yoshikazu Kanazawa $\cdot$ Ryuji Ohashi $\cdot$ \\ Tadashi Machida $\cdot$ Yasutomo Suzuki · Takayuki Aimoto • \\ Kaoru Kubota $\cdot$ Akihiko Gemma
}

Received: 24 June 2013/Accepted: 1 July 2013/Published online: 7 August 2013

(C) The Japan Society of Clinical Oncology 2013

\begin{abstract}
Metastatic spermatic cord tumors are relatively rare, and gastric, colorectal, and pancreatic cancers have been reported as the primary lesion $[1,2]$. We experienced a case of metastasis to the spermatic cord 1 year after surgery for gastric cancer. A 68 -year-old male developed a painful right inguinal mass 1 year after surgery for gastric cancer and tubular adenocarcinoma was diagnosed by aspiration biopsy cytology from the right inguinal mass. A solitary inguinal metastasis was suspected by FDG-PET, and laparoscopic tumor resection was performed for definite diagnosis. Postoperatively, spermatic cord metastasis of gastric cancer was diagnosed pathologically by hematoxylin and eosin staining and immunohistochemistry (CK7+ and CK20-) of the resected samples. As to the metastasis route to the spermatic cord, development from
\end{abstract}

This case report was accepted for publication by the Journal of Nippon Medical School and is scheduled to be published [3]. The report was used for a case conference in the journal after approval was obtained in advance.

M. Seike $(\bowtie) \cdot$ K. Kubota · A. Gemma

Department of Pulmonary Medicine and Oncology, Graduate

School of Medicine, Nippon Medical School, Tokyo, Japan

e-mail: mseike@nms.ac.jp

Y. Kanazawa · T. Aimoto

Department of Surgery, Nippon Medical School, Tokyo, Japan

R. Ohashi

Division of Diagnostic Pathology, Nippon Medical School,

Tokyo, Japan

T. Machida

Department of Radiology, Nippon Medical School, Tokyo, Japan

Y. Suzuki

Department of Urology, Nippon Medical School, Tokyo, Japan minimal peritoneal disseminated nodules was suspected. Metastatic routes were speculated by comprehensive analysis of clinical information such as laparoscopic findings and a pathohistological approach including immunohistochemistry.

Keywords Stomach cancer - Spermatic cord tumor . Metastasis

\section{Case presentation}

Dr. Kubota (medical oncologist, conference chairperson)

Good evening everyone. Welcome to the Cancer Board Conference.

Today, we would like to discuss a case of metastatic spermatic cord tumor from stomach cancer 1 year after curative gastrectomy. Dr. Kanazawa, please begin the case presentation.

\section{Dr. Kanazawa (gastrointestinal surgeon)}

The patient was a 68 -year-old male. As to his past history, he underwent Bassini's operation for right inguinal hernia at 48 years of age. In addition, he was diagnosed with advanced gastric cancer at 67 years of age and underwent distal gastrectomy with D2 lymphadenectomy. Pathological examination of the resected specimen revealed stage IIIA and postoperative adjuvant chemotherapy with TS-1 was given. One year after the surgery, a painful right inguinal mass appeared just below the scar of the hernia operation. Abdominal CT demonstrated a mass of $4 \mathrm{~cm}$ in size at the right groin (Fig. 1) and aspiration biopsy 


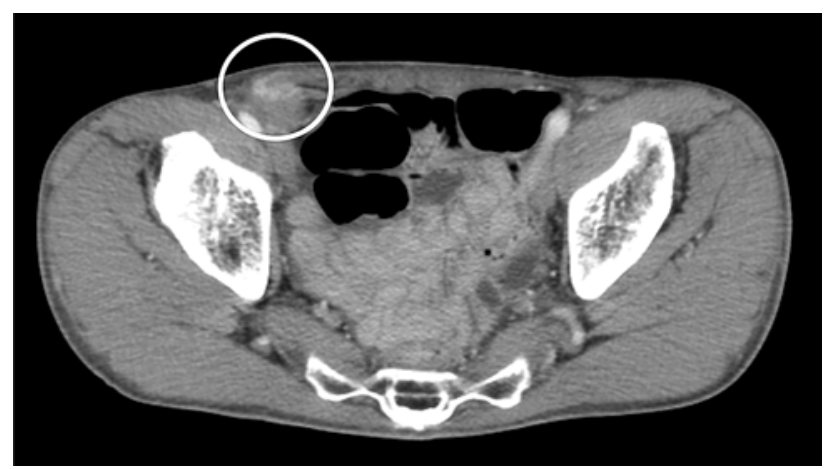

Fig. 1 Abdominal CT detected a $4 \mathrm{~cm}$ tumor in the right inguinal region

cytology revealed tubular adenocarcinoma. FDG-PET showed FDG accumulation at the right groin but no abnormal accumulation at the intraperitoneal lymph nodes, including paraaortic lymph nodes, or other intraperitoneal organs. Colonoscopy revealed no abnormal findings, and there was no abnormality in the urological system. Tumor markers such as CEA, CA19-9, and STN were not elevated.

\section{Dr. Kubota}

At this point, what was your differential diagnosis of the right inguinal tumor?

\section{Dr. Kanazawa}

Recurrence was suspected at first, because he had undergone surgery for gastric cancer. However, there was no imaging finding of hematogenous metastasis, lymphogenous metastasis, or peritoneal dissemination other than the right inguinal tumor. The tumor was not typical for metastasis of gastric cancer.

\section{Dr. Kubota}

As to the solitary right inguinal tumor, what do you think about the possibility of inguinal lymph node metastasis from a urological malignant tumor?

\section{Dr. Suzuki (urologist)}

In general, prostatic cancer and bladder cancer metastasize to intrapelvic lymph nodes and they rarely metastasize to inguinal lymph nodes. Moreover, penile cancer is a urological tumor that may metastasize to inguinal lymph nodes, but there was no remarkable finding in the penis and metastasis from penile cancer was unlikely in this case.

\section{Dr. Kubota}

What do you think about the possibility of metastasis to inguinal lymph nodes from a perianal tumor?

Dr. Aimoto (gastrointestinal surgeon)

Anal cancer can metastasize to inguinal lymph nodes, but the location of the inguinal lymph nodes is, in general, too high. In fact, colonoscopy revealed no abnormal findings.

\section{Dr. Kubota}

Then, as a radiologist, what is your point of view?

\section{Dr. Machida (radiologist)}

Findings of imaging modalities such as PET showed no evidence of a malignant tumor other than the right inguinal malignancy. When metastasis to inguinal lymph nodes is diagnosed, solitary metastasis to the groin without systemic hematogenous or lymphogenous metastasis is rare. As Dr. Suzuki mentioned, the location was too high for metastasis to inguinal lymph nodes and I thought that it was unlikely.

\section{Dr. Kubota}

Dr. Kanazawa, is solitary inguinal lymph node metastasis like this typical for a recurrence pattern of gastric cancer?

\section{Dr. Kanazawa}

There was a report of metastasis to inguinal lymph nodes as one of multiple lymph node metastases, but there was no report of solitary metastasis like this. Since there was no other malignant tumor by imaging modalities, the diagnosis before surgery was inguinal lymph node metastasis from gastric cancer.

\section{Dr. Kubota}

Next, Dr. Kanazawa, could you report the results of inguinal mass resection and histopathological examination of the resected specimen?

\section{Dr. Kanazawa}

First, I would like to report the images of gastric cancer and pathological findings from 1 year before. The gastric cancer was type 3 moderately differentiated adenocarcinoma of $4 \times 4 \mathrm{~cm}$ in size and located in the middle of the gastric body. Metastasis was positive in 2 of 47 lymph nodes and T4aN1M0 stage IIIA was diagnosed. Immunohistochemistry revealed 


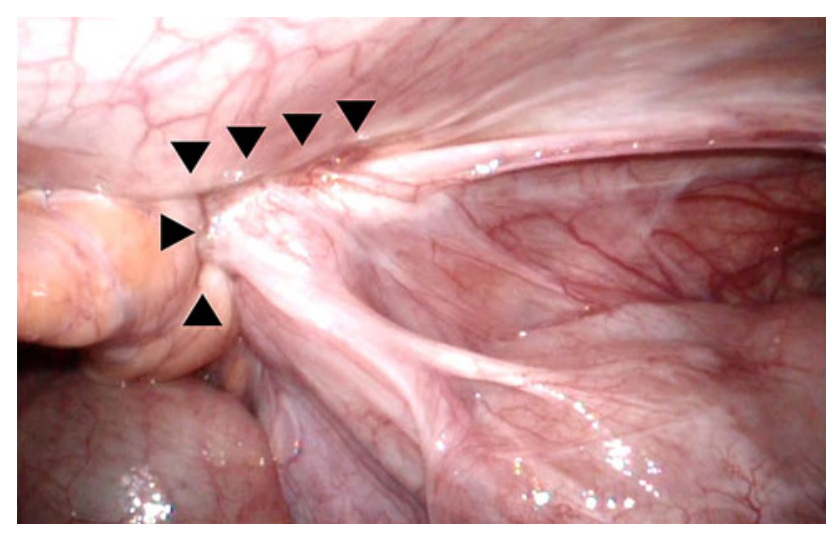

Fig. 2 Invagination of the peritoneum with small nodules was detected from the median umbilical fold to the lateral umbilical fold

positivity for CK7 and negativity for CK20, consistent with gastric cancer.

Since recurrence of gastric cancer via peritoneal dissemination could not be ruled out, laparoscopy was performed first. There was no clear finding suggesting recurrence such as peritoneal dissemination, liver metastasis, and lymph node metastasis in the peritoneal cavity. In addition, peritoneal lavage cytology was negative. Therefore, solitary recurrence was suspected and inguinal mass resection was carried out. Laparoscopy revealed indentations of the peritoneum from the medial umbilical fold to the lateral umbilical fold, and the tumor was located at the dorsal side of the indentations (Fig. 2).

\section{Dr. Ohashi (pathologist)}

Pathologically, moderately differentiated adenocarcinoma resembling the gastric cancer histology previously observed in the same patient was recognized in a scattered manner in the stroma around the spermatic cord. Immunohistochemistry revealed positivity for CK7 and negativity for CK20, consistent with the staining pattern of the primary gastric lesion. Taken together, gastric cancer metastasis to the spermatic cord was diagnosed (Fig. 3a). The sample collected from the abdominal wall showed infiltration of cancer cells with stromal proliferation. Immunohistochemistry of the same sliced specimen showed a ring of scattered peritoneal mesothelial cells positive for calretinin (Fig. 3b). It was presumed that cancer infiltration at this site induced complicated indentations of the peritoneum and eventually spermatic cord invasion developed from disseminated nodules.

\section{Dr. Kubota}

Metastasis was observed only at the right spermatic cord in the present case. More interestingly, the mass appeared just below the scar of the inguinal hernia operation performed
20 years before. What do you think about the relationship with the hernia operation?

Dr. Aimoto

Bassini's operation was carried out for inguinal hernia 20 years before. Generally, no operation scar is observed on the peritoneal wall by laparoscopy in such cases. In the present case, the indentations of the peritoneum were markedly abnormal findings and so-called minimal peritoneal metastasis was suspected. It was presumed that minimal disseminated nodules, by chance, developed on the peritoneal wall between the medial and lateral umbilical folds and formed a mass at the groin along the spermatic cord. I think that this hypothesis was corroborated by the detailed pathological exploration.

\section{Dr. Ohashi}

Dr. Aimoto used the phrase "by chance", but was it appropriate? Pathologically, neovascularization often occurs at the scar after surgery and consequently a new bloodstream network and collaterals are created. In the present case, hematogenous metastasis via this route may have occurred. In this context, tumor formation just below the operation scar may be significant.

\section{Dr. Kubota}

Is there any doctor who has experience in recognizing metastasis at the operation scar?

\section{Dr. Kanazawa}

We often experience cancer recurrence at the site of port placement for laparoscopic surgery.

Dr. Aimoto

Although I have no such clinical experience, tumors often developed at the wound of laparotomy in carcinogenesis experiments in animals. As Dr. Ohashi mentioned, if the patient had not undergone surgery for inguinal hernia, there would have been no recurrence at this site and the recurrence pattern would have been different.

\section{Dr. Kubota}

Lastly, could you report the clinical course thereafter?

\section{Dr. Kanazawa}

Since the possibility of a residual tumor was considered, low-dose oral TS-1 chemotherapy was given in addition to 

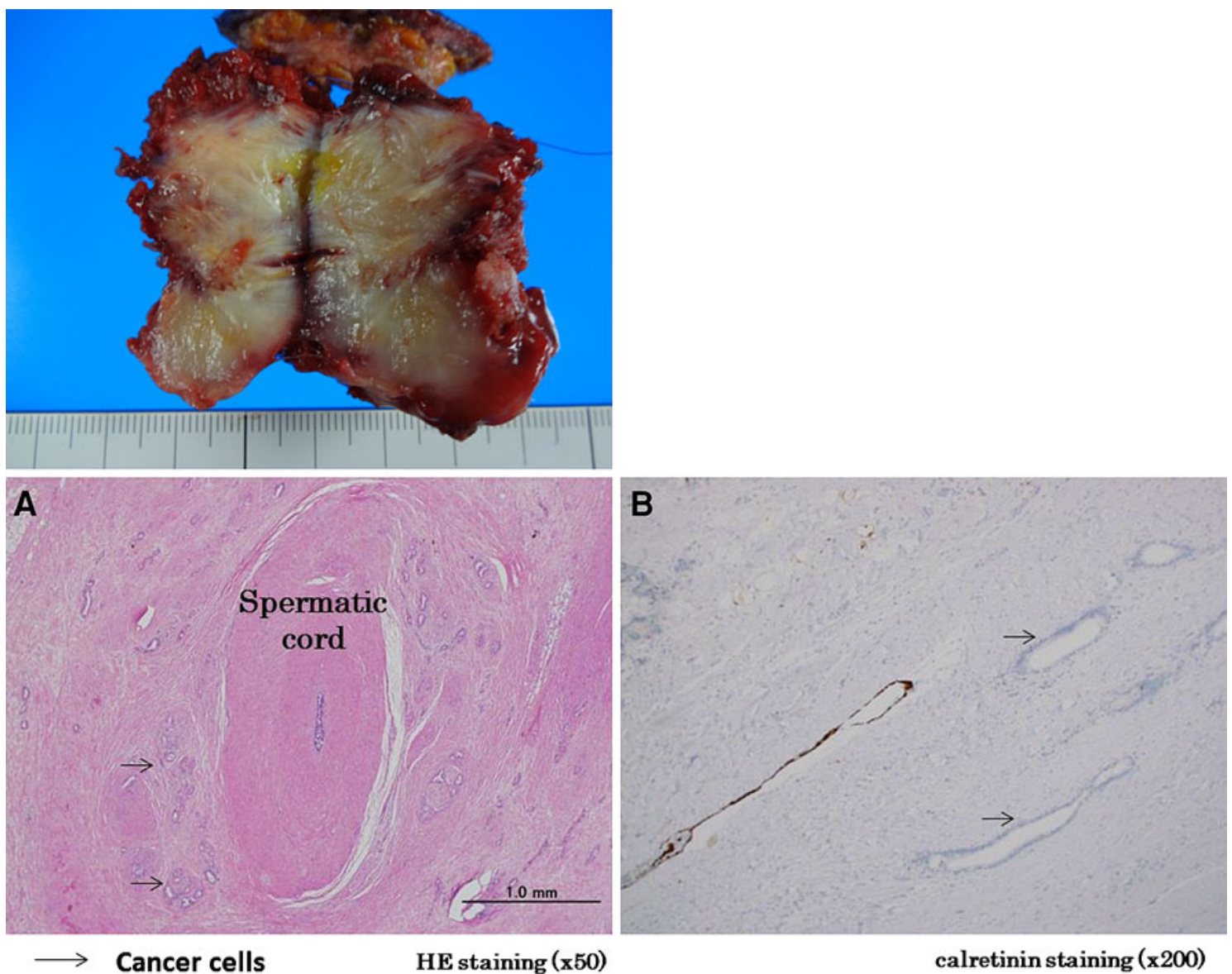

Fig. 3 a Moderately differentiated tubular adenocarcinoma was diffusely infiltrated throughout the connective tissue surrounding the spermatic cord (HE staining, $\times 50$ ). b Immunohistological findings

local radiation therapy. Later, biweekly CPT-11 therapy was added, but unfortunately, ascites and new peritoneal disseminated nodules appeared.

\section{Dr. Kubota}

Although TS-1 was used for postoperative adjuvant chemotherapy after the first surgery and recurrence was observed, you added TS-1 chemotherapy to radiation therapy. Could you explain the reason for using TS-1 again?

\section{Dr. Kanazawa}

Although evidence had not been established, I administered TS-1 with the expectation that it would sensitive the residual tumor to radiation therapy.

\section{Dr. Kubota}

Are there any questions? Thank you for attending this conference today.

show that mesothelial cells (arrows) were positive for calretinin (calretinin staining, $\times 200$ )

What we learned from this case conference

1. We reported our experience with the diagnosis and treatment of a rare case of metastasis to the spermatic cord from gastric cancer.

2. Development from minimal peritoneal disseminated nodules was suspected as the most likely metastasis route to the spermatic cord. The metastasis route was speculated by comprehensively examining clinical information such as laparoscopic findings in combination with a pathohistological approach including immunohistochemistry for calretinin.

Conflict of interest Honoraria: K. Kubota and A. Gemma, Taiho Pharmaceutical Co. Ltd.; A. Gemma received research funding from Taiho Pharmaceutical Co. Ltd. Others declare no conflicts of interest.

\section{References}

1. Algaba F, Santaularia JM, Villavicencio H (1983) Metastatic tumor of the epididymis and spermatic cord. Eur Urol 9:56-59 
2. Ota T, Shinohara M, Tanaka M et al (2000) Spermatic cord metastases from gastric cancer with elevation of serum hCG-beta: a case report. Jpn J Clin Oncol 30:239-240
3. Kanazawa Y, Kato S, Fujita I et al (2013) Spermatic cord tumor metastatic from stomach cancer 1 year after curative gastrectomy. J Nippon Med Sch (in press) 